VII.

\title{
Ueber die Einwirkung des Phosgens auf den menschlichen und thierischen Körper.
}

\author{
Von
}

Dr. med. et phil. Robert Müller, Elberfeld.

Ueber die toxikologischen Wirkungen des Phosgens liegt bisher eine zusammenfassende und eingehendere Untersuchung nicht vor, obgleich dieser Stoff einerseits in der chemischen Industrie in zicmlich beträchtlichen Mengen verbraucht wird, andererseits das Phosgen als Spaltungsproduct des Chloroforms als Ursache der Nachkrankheiten der Chloroformnarkose in Betracht kommt. Mehrere am Menschen beobachtete Vergiftungen, bei denen allerdings neben Phosgen noch Phosphoroxychlorid einwirkte, waren der Anlass, diesen Körper eingehender auf seine toxikologische Wirkung zu untersuchen.

Das Phosgen, $\mathrm{COCl}_{2}$, dasselbe wie Chloroformylchlorid, ist eine erstickend riechende Flüssigkeit vom Siedepunkt $8,2^{\circ}$ (corrigirt) und dem specifischen Gewicht 1,432 bei $0^{\circ}$. Es ist leicht löslich in Benzol, Eisessig und den meisten flüssigen Kohlenwasserstoffen. Von kaltem Wasser wird es nur sehr langsam zersetzt, sehr rasch dagegen von heissem unter Bildung von $\mathrm{CO}_{2}$ und $\mathrm{HCl}$. In Alkohol löst es sich unter Bildung von Chlorameisensäureester, trot $\%$ der symmetrischen Formel $\mathrm{Cl}-\mathrm{CO}-\mathrm{Cl}$ wird doch nur ein Chloratom gegen $-\mathrm{OC}_{2} \mathrm{H}_{5}$ ausgewechselt. Metalle, wie $\mathrm{K}$, $\mathrm{Zn}$, Sn, As und Sb entzichen ihm bei höherer Temperatur das Chlor, mit Schwefelcadmium entsteht bei $270^{\circ} \mathrm{COS}$. Mit $\Lambda$ mmoniak bildet es Ammoniumchlorid, Harnstoff und kleine Mengen Guanidin und Cyanursäure.

Es bildet sich aus $\mathrm{Cl}_{2}$ und $\mathrm{CO}$ direct unter dem Einflusse des Sonnenlichtes (Davy), zweitens wenn man $\mathrm{CO}$ und $\mathrm{CCl}_{4}$ bei $400^{\circ}$ über Bimstein leitet (Schützenberger 1868), drittens aus Tetrachlorkohlenstoff und Phosphorpentoxyd bei 200-210 ${ }^{0}$ [Gustavson 1871] ${ }^{1}$ ).

Das wichtigste Anwendungsgebiet des Phosgens in der chemischen Industrie liegt in der Farbstofffabrication und zwar sind es die Diphenylnaphthylmethanfarbstoffe und eine Reihe von Azofarbstoffen, bei deren Herstellung es benutzt wird.

1) Vergl. Beilstein, Organische Chemie. III. Aufl. Bd. I. S. 546. 
Für dic Diphenylnaphthylmethan-Farbstoffe dient es zur Herstellung des Michler'schen Ketons, des Tetramethyldiaminobenzophonons

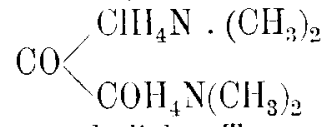

indem Dimethylanilin bei gewönlicher Temperatur mit der theoretischen Nenge Phosgen gesättigt und das Reactionsproduet mit Wasser gefält wird, der Nicderschlag wird dann aus Alkohol umkrystallisirt ${ }^{1}$. Durch kinwirkung ron Phenyl-e-naphthylamin auf 'Tetramethyldaminobenzophenonchlorid entsteht der von Caro und Kern 1883 gefundene Farbstoff, der als Vietoriablau in den Handel gebracht wird, dahin gehört weiter der von Nastrogel 1892 dargestellte Farbstoff, das Neuvictoriablau R, ferner das Nachtblau, das Victoriablau $4 \mathrm{R}$ und das Wollgrün $\mathrm{S}^{2}$ ).

Line zweite Gruppe von Farbstoffen, zu deren Herstellung das Phosgen in der Industrie benutzt. wird, sind gewisse 1 zofarbstoffe, welchen die 2,5-Amidonaphthol-7-Sulfosäure zu Grunde liegt und die als Benzoechtorange $S$ und Benzoscharlachmarken in den Handel gebracht werden ${ }^{3}$ ); zu dieser Farbstolfgruppe gehört auch das Baumwollgelb G, das Natriumsalz der Diamidodiphenylharnstoffdiazobisalicylsüure, welches auch unter direeter Benutzung des Phosgens hergestellt werden kann ${ }^{4}$ ).

Ein weiteres Anwendungsgebiet des Phosgens in der chemischen Industric liegt in der Herstellung gewisser pharmaceutischer Producte und zwar sind es solche von erleblicher Wiehtigkeit, bei denen es verwandt wird. Vinerscits sind es Kohlensäureester, von denen des Duotal, Creosotal, das Blenal, das Mristochin und Euchinin die wichtigsten sind und andererseits sind es gewisse Urethanabkömmlinge, wie das Hedonal, deren Herstellung über dic Chlorocarbonate geht ${ }^{5}$ ).

Welche Bedeutung der Phosgenverbrauch in der lechnik hat, geht daraus hervor, dass eine der grössten deutschen chemischen Fabriken, welche aber im Phosgenverbrauch keineswegs an erster Stelle steht, im Jahre 1906 etwa $40000 \mathrm{~kg}$, im Jahre 1907 etwas über $35000 \mathrm{~kg}$ und im ersten Halbjahr 1908 etwa $18500 \mathrm{~kg}$ verbrauchte.

Was die Beobachtungen am Menschen anbetrifft, so standen mir drei Fälle zur Verfügung, welche sich die Intoxication in der Farbstofffabrication zugezogen hatten. Es bot sich mir Gelcgenheit, diese Fälle selbst des öfteren zu untersuchen, ich verdanke aber das vollständige Material dem Entgegenkommen des I. Arztes der Fabrik und der betreffenden Section der chemischen Berufsgenossenschaft. Keiner dieser Fälle stellt eine reine Phosgenvergiftung dar, da neben der Einwirkung von Phosgen diese Personen anch der von Phosphoroxychlorid, das bei der Weiterverarbeitung des Mlichler'schen Ketons zur Farbstoffabrication vielfach

1) Ebendas. Bd. IlI. S. 185 .

2) Gustar Schulz und Paul Julius, 'labellarische Uebersicht der im Handel befindlichen kïnstlichen organischen Farbstoffe. 4. Aufl. 1902. S. $176 \mathrm{fl}$.

3) Friedlaender, Fortschritte der 'l'heerfarbenfabrikation 1900-1902. Bd. 6, S. 954 .

4) Gustav Schulz und Paul Julius. 1. c. S. 74.

5) Fr. Iofmann, Ueber Studien in der Kohlensäurereihe. Zeitschr, für angewandte Chemie. XXI. II. 38. S. 1986 fr. 
benutzt wird, ausgesetzt waren. Der Verlauf der Intoxication und ihrer Folgen geht aus den folgenden Krankengeschichten hervor:

Fall I. Der damals 46 jährige, als Betriebsschlosser beschäftigte Arbeiter athmete am 30. 8. 1906 bei der Entfernung des Abdruckrohres aus dem Ketonschmelzkessel geringe Mengen Phosgen und $\mathrm{POCl}_{3}$ ein, zusammen mit den beiden anderen Personen, auf wolche sich die folgenden Kranliengeschichten beziehen. Fr war schon seit Jahren in jenem Betriebe beschäftigt und hatte bereits wiederholt unerhebliche Mengen von $\mathrm{POCl}_{3}$, und $\mathrm{COCl}_{2}$ eingeathmet, welche aber nicht zu ernstlicheren Störungen seines Befindens geführt hatten, indessen latte der Unfall vom 30. 8. allInählich derartig sich steigernde Beschwerden zur Folge, dass er sich am 11. 10. 1906 lirank meldete und für arbeitsunfähig befunden wurde. Die eingehende Untersuchung am 7. 12. 1906 ergab folgenden Befund: Es ist ein mittelkräftiger, mittelmässig gut genährter Mann mit etwas blasser Gesichtsfarbe und blassen Schleimhäuten. Der Thorax ist normal grebaut, beide Hälften heben sich gat und gleichmässig bei tiefer Athmung, die Athmung zeigt in der Ruhe normales Verbalten. Der Puls ist klein, etwas hart, gespannt und träge, 60-70 Schläge in der Minute. Der Herzspitzenstoss ist verbreitert und innerhalb und ausserhalb der Brustwarzenlinie im V. Zwischenrippenraum fühlbar. Ueber sämmtlichen Ostien des Herzens hört man ein langgezogenes systolisches blasendes Geräusch, ebenso über der Carotis am Halse. Der zwoite Ton über der Aorta ist kaum hörbar, ebenso nicht der erste Mitralton. Die absolute Herzdämpfung reicht nach links bis zur Brustwarzenlinie, nach rechts ist sie nicht vergrössert, sio reicht bis zum linken Sternalrand. Die Breite der relativen Dämpfung im 4. Intercostalraum beträgt $17-18 \mathrm{~cm}$. Der Blutdruck $=120 \mathrm{~mm}$ (Riva-Rocci) ist erniedrigt. Die Lungengrenzen sind vorn lechts der untere Rand der 4. Rippe, es besteht keine abnorme Dämpfung. Das Athemgeräusch ist vesiculär and besonders in den unteren Partien begleitet von meist trockenem Rasseln. Der Harn ist frei von abnormen Bestandtheilen, Leber und Milz sind nicht vergrössert, nervöse Störungen fehlen. Er klagt über ständiges Herzklopfen, Schmerzen in der Herzgegend und Luftmangel bei schnellem Gehen und Bergsteigen, ferner über Husten und Auswurf, der zeitweilig Blut enthält, sowie über allgemeine Schwäche. In letzter Zeit fühlte er sich wohler und bemerkt namentlich eine Besserung der Herzbeschwerden.

Der Erkrankte ist seit Jahren bekannt, wiederholt aus anderen goringfügigen Anlässen behandelt und untersucht worden, zum letzten Male am 3. 7. 1905, wo er eine kleine Menge Phosgen eingeatbmet hatte und infolge dessen an Athembeschwerden litt, wolche am 7.7. 1905 vollständig beseitigt waren. Dio Organe der Brust, speciell das Herz, erwiesen sich damals als völlig gesund. Es ist anzunehmen, dass die Erkrankung, welche eine Hypertrophie des linken Herzens mit Insufficienz und Stenose der Aortenklappe darstelit, auf die Intoxication zurieckzuführen ist; wenn auch die Anfänge der Herzerkrankung wohl schon längere Zeit zurückliegen und vielleicht auf die wiederholte Einwirkung der Schädigungen durch $\mathrm{COCl}_{2}$ und $\mathrm{POCl}_{3}$ in kleinen Mengen zurückgeführt werden können, so trat doch eine erhebliche Verschlimmerung, welche schliesslich die völlige Arbeitsunfähigkeit bedingte, im Anschluss an den erwähnten Unfall ein. Bei entsprechender allgemeiner und symptomatischer Behandlung fand eine fortschreitende Besserung statt und der Erkrankte konnte vom 11.3. 1907 an seine Arbeit wieder aufnehmen.

Bei der Entlassung aus der Behandlung (26.3.07) bestand noch etwas Husten, Herzklopfen und Athembeschwerden stellten sich nur noch bei liörperlichen Anstrengungen ein. Die absolute Herzdämpfung reicht nach links nicht ganz bis zur Mamillarlinie, nach rechts bis zum linken Brustbeinrande, die relative Herzdämpfung zeigt eine Breite von $16 \mathrm{~cm}$ im IV. Intercostalraume. Der erste Ton an der Herzspitze und über allen Arterien ist noch stark blasend, der zweite Aortenton ist jetzt hörbar, der Puls ist mittelkräftig, ziemlich voll und regelmässig, 60-66 Schläge in der Minute. Die lungengrenzen sind normal und gat verschieblich, es findet sich keine abnorme 
Dämpfung über den Lungen, das Athemgeräusch ist vesiculär, links hinten unten noch etwas rauh und begleitet von spärlichem trockenen Rasseln. Der Auswurf ist schleimig und enthält zeitweilig ein wenig Blut, doch fiel die öfters wiederholte Untersuchung auf Tuberkelbacillen negativ aus. Das Aussehen ist befriedigend, die Schleimhäute zeigen noch eine leicht trübe Färhung, Abdominalorgane 0. B.

In den folgenden Jahren besserte sich die chronische Bronchitis zeitweilig, manchmal verschlimmerte sie sich; es entwickelte sich auf ihrem Boden eine indurirende Tuberkulose, welche aber fast stationär blieb, so dass der Mann bis jetzt dauernd arbeitsfähig war ${ }^{1}$ ).

Fall II. Der damals 38jährige, schon seit mehreren Jahren im Betrieb beschäftigte Arbeiter, liam bereits am 3. 9. 06, also am 5. Tage nach dem Unfall, in Behandlung und war damals der Befund folgender: Es bestehen die Erscheinungen einer schweren Bronchitis, reichlich feine feuchte und trockene Rasselgeräuscbe, daneben stärkeres Emphysem, vorne rechts reicht die Lunge bis zur VIII. Rippe, es bestehen erhebliche $\Lambda$ thembeschwerden und stärkerer Husten. Am Herzen ist eine Dilatation des linken Ventrikels nachweisbar, die Herzaction ist unregelmässig und stark beschleunigt, es besteht mässiges Fieber.

An 7. 12.06 giebt das Attest das folgende Bild: Es ist ein schwächlich gebauter, wenig gut genährtar Mann. Die Gesichtsfarbe ist geröthet und etwas cyanotisch, die Schleimhäute sind ebenfalls mit Blut überfüllt, der Brustkorb ist abgeflacht und fassförmig erweitert. Der Herzspitzenstoss ist verbreitert und inner- und ausserhalb der Mamillarlinie fühlbar und sichtbar. Die absolute Herzdämpfung reicht nach oben bis num oberen Rande der IV. Rippe, nach rechts bis zum linken Brustbeinrand, vielleicht ein wenig darüber hinaus, nach links reichlich bis zur Mamillarlinie. Die Breite der relativen Herzdämpfung beträgt im IV. Intercostalraume 17 bis $18 \mathrm{~cm}$. Die Herztöne sinả laut und scharf accentuirt, der Blutdruck ist niedrig $(12 \mathrm{~cm}$ mit dem Riva-Rocei'schen Apparat bestimmt). Der Puls ist stets stark beschleunigt, zählt 100-120 Schläge, er ist gespannt und unregelmässig. Auch in der Ruhe besteht leichte inspiratorische Athemnoth. Dic Lungengrenze ist rorne rechts die VII. Rippe, hinten beiderseits die XII. Rippe. Es besteht keine abnorme Dämpfung über den Lungen. Besonders hinten unten hört man raubes verschärftes Athmen und meist trockenes Rasseln, im Uebrigen ist die Athmung überall vesiculär. Der Auswurf ist zäh, schleimig, enthält keine geformten Bestandtheile und keine Tuberkelbacillen, ist zeitweilig mit Blut vermischt, das aber wohl aus dem Rachen stammt. Die Leberdämplung überragt einfingerbreit den Rippenbogenrund. Die Leber fühlt sich glatt, etwas hart an. Milz, nicht nachweisbar vergrössert, keine nervösen Störungen, der Harn ist frei von abnormen Bestandtheilen. Er giebt an, ständig an $\Lambda$ themnoth und Hustenreiz zu leiden, er klagt ferner über Herzklopfen und Stiche in der Ilerzgegend. In der Nacht habe er sehr häufig Anfälle von grosser Athemnoth, Brustbeklemmungen und Unruhe.

Der Patient hatte in den vorbergehenden Jabren mehrmals $\mathrm{COCl}_{2}$ und $\mathrm{POCl}_{3}$ eingeathmet und im Anschluss hieran wiederholt an Entzündungen der Athmungsorgane gelitten. Auch eine Vergrösserung des Herzens nach links war schon früher, wenn auch in geringerem Grade nachwcisbar. Dagegen sind die zur Zeit (7. 12. 06) vorliegenden Erscheinungen auf den Unfall vom 30. 8. 06 zurückzuführen und erst neuerdings ist eine Vergrösserung der Leber nachweisbar. Es besteht nun neben schwerer chronischer Bronchitis und Emphysem eine Herzhypertrophie, vorwiegend nach links, eine funktionelle Insufficienz der Herzklappen und eine Vergrösserung der Leber.

Die Krankheit zog sich sehr in die Länge und der Patient wurde am 6. 6. 07 zum ersten Male aus der Behandlung entlassen. Das Heilverfahren wurde wiederholt

1) Zusatz bei der Correctur: Der Arbeiter ist am 8.1 .11 gestorben, nachdem er vom 29. 12. 10 an arbeitsunfähig war. 
durch Lungenblutungen, durch Entstehung von Furunkeln und zuletzt durch eine Parotitis epidemica unterbrochen. 'Trotz der Schwere der vorliegenden organischen Läsionen war das erreichte Resultat vorübergehend ein recht günstiges. Die Besserung dauerte indessen nicht lange, seit dem 18.7.07 war Patient wieder arbeitsunfähig und wurde einem Krankenhause in Bonn überwiesen, wo er von Herrn Prof. Rumpf eingehend untersucht und begutachtet wurde. Dieses Gutachten enthält auch die Angaben des Patienten unmittelbar nach seinem Unfall: Er habe sofort starke Beklemmungen auf der Brust bemerkt, keine Luft mehr bekommen können und sei mit den beiden anderen Arbeitern an das Fenster gestürzt. Die Athemnoth habe sich immer noch gesteigert und es seien ihm Schleim und Schaum aus dem Munde gekommen. Diese Beschwerden seien erst gegen Abend etwas besser geworden. In der folgenden Nacht habe er wieder derartige $\Lambda$ thembelicmmungen gehabt, dass er immer wieder zum Fenster habe gehen müssen, um frische Luft zu schnappen; am nächsten Tage habe er wieder versucht zu arbeiten, habe aber ständig unter Kurzathmigkeit zu leiden gebabt.

Aus dem Gutachten ist ferner zu erwähnen, dass sich im Harn etwas Eiweiss fand, der Hämoglobingehalt betrug 85 pCt, eine Hyperleukocytose bestand nicht. Fine weitere Untersuchung rom 24.1.08 in demselben Krankenhause ergab eine weiter fortschreitende Besserung, indem die Herzdämpfung annähernd normale Grösse hatte und der Harn wieder eiweissfrei war, dagegen bestand noch eine geringe Schmerzhaftigkeit und Vergrösscrung der Leber.

Der Patient entwickelte sich nun in der folgenden Zeit in recht ungünstiger Weise, er trank ziemlich viel, klagte trotz leichter $\Lambda$ rbeit über alle möglichen Beschwerden, und that seine Arbeit nur schlecht und unregelmässig. Dies führte dazu, dass er wieder dem Krankenhause in Bonn überwiesen wurde und dort ein weiteres Attest über ihn ausgestellt wurde. Aus diesem erscheint Folgendes erwähnenswerth: die Lungengrenzen sind nicht erweitert, eine Dämpfung ist nicht vorhanden, das Athemgeräusch ist überall vesiculär. Er hustet ziemlich viel und hat zuweilen etwas grünlich-gelblichen Auswurf. Die Herzämpfung zeigte geringe Verbreiterung nach links, der Spitzenstoss ist ausserbalb der Mamillarlinie za fühlen. Diese Yergrösserung des Herzens wird durch eine orthodiagraphische Röntgenaufnabme bestätigt. Die Herztöne sind rein, Puls 68--72 Schläge in der Minute. Die Blutdruckmessung nach Riva-Kocci ergiebt schwache Wiederkehr des Pulses bei 174-178, starke bei $120 \mathrm{~mm}$ Hg-Druck. Die Leber ist nicht mehr vergrössert, der Leberrand ist auf Druck noch schmerzhaft. Der Urin enthält geringe Spuren von Eiweiss, die mikroskopische Untersuchung des Harnsediments ergiebt spärliche Mengen von Fetttröpfchencylindern. Im Laufe der Zeit besserten sich die Erscheinungen von Seiten des Herzens und der Nieren zeitweilig, dagegen verschlimmerte sich der Lungenbefund spontan beträchtlich. Der Patient hat sich zum völligen Unfallneurastheniker entwickelt, der nicht mehr arbeitet.

Fall III. Der Patient ist Aufseher in einem Farbstoff betrieb und erlitt denselben Unfall wie die beiden vorhergehenden Personen. Er verspürte bereits am folgenden Tage Athembeschwerden, wie solches bei ähnlichen Gelegenheiten schon öfters vorgekommen war, die aber jedesmal rasch vorübergegangen waren. Der Erkrankte kam bereits am 31. 8. 06 in Behandlung, der Befund war damals folgender: Ueber den mittleren und unteren Parthien der Lunge, und zwar vorwiegend rechts, hört man mittel- und kleinblasiges Rasseln, Pfeifen, Giemen und rasehes Athmen. Puls 100, etwas klein und regelmässig. Zeichen einer Lungenblähung bestehen nicht, es ist leichter Hustenreiz und leichte inspiratorische Dyspnoe vorhanden. Fr arbeitete zunächst weiter, als aber seine Beschwerden sich verschlimmerten, musste er am 11. 10. 06 die Arbeit aussetzen. Gegen den 20.10. 06 stellten sich erhebliche Herzbeschwerden ein, mit stark beschleunigtem und unregelmässigem Puls. Am 5. 12.06 war der Befund folgender: Der Patient ist gut genährt, kräftig, und sieht nicht be- 
sonders kränklich aus. Die Gesiehtsfarbe ist geröthet und ein wenig cyanotisch. Der Puls ist stets stark beschleunigt, zählt etwa 120 Schläge in der Minute, ist unregelmässig und ziemlich klein uud leicht unterdrückbar. Die Athmung ist etwas angestrengt, sehr oberllächlich und bei Bewegungen beschleunigt. Die Lungengrenzen sind ein wenig nach unten verschoben, vorne rechts uuterer Rand der VI. Rippe, hinten XII. Rippe beiderseits, aber bei tiefer Respiration gut verschieblich. Das Athemgeränsch ist leise, vesiculär, beiderseits etwas rauh. Ueber beide Lungen vertheilt hört man feines trockenes und auch feuchtes Rasseln, hauptsächlich am Ende des Inspiriums; besonders vorne rechts über dem Mittellappen starkes Schnurren. Der Herzspitzenstoss ist innerbalb der Brustwarzenlinie an normaler Stelle schwach fühlbar. Die absolute Dämpfung reicht nach rechts bis etwas über den linken Brustbeinrand hinaus, nach links ist dieselbe kaum verbreitert. Die relative Dämpíng ist entschieden verbreitert, sie misst im IV. Intercostalraum $16 \mathrm{~cm}$. Die Herztïne sind sehr leise aber rein. Die Leber ist nicht vergrössert und ihr Rand nicht fühlbar, die Gegend derselben ist etwas druckempfindlich, die Milz ist nicht vergrössert. Nervöse Störungen bestehen nicht. Der Blutdruck ist ein sehr niedriger; gemessen mit dem Riva-Rocci'schen Apparat $110 \mathrm{~mm}$. Der Urin ist klar und reagirt sauer, er entbält zeitweilig Spuren ron Eiweiss, sonst keine abnorme Bestandtheile. Die subjectiven Beschwerden bestehen in ständiger Kurzathmigkeit, Herzklopfen und stechenden Schmerzen in der Herzgegend, besonders bei Bewegungeu, und in Hustenzeiz. Der Auswurf ist sehr spärlich and schleimig. Zeitweilig finden sich Schmerzen in der Lebergegend.

Der Befund besserte sich fortschreitend, der Puls warde kräftiger und weniger beschleunigt, die Breite der relativen IIerzdämpfung betrug am 28. 1. 07 nur noch $14-15 \mathrm{~cm}$, der Blutdruck, nach Riva-Rocoi bestimmt, $12-13 \mathrm{~cm}$. Auch die liatarrhaliscben Erscheinungen seitens der Athmungsorgane haben erheblich abgenommen, Eiweiss fand sich seit längerer Zeit nicht mehr im Harn. Am 22. 3.07 konnte er aus der Behandlung entlassen werden. Aus dem Entlassungsbefund sei Folgendes erwähnt: „Der Puls zählt in der Ruhe 80-90 Schläge, ist jedoch ziemlich lräftig und regelmässig. Die Athmung ist in der Ruhe frei und nicht beschleunigt, es besteht noch mässiger Hustenreiz und oberflächliches Räuspern. Der Auswurf ist spärlich und schleimig. Die Lungengrenzen sind vorn rechts der obere Rand der VI. Rippe, hinten beiderseits die XI. Rippe, die Grenzen sind bei tiefer Athmung gut verschieblich. Vorn rechts üher dem Schlüsselbein und hinten unten beiderseits bört man rauhes Athmen, feines Rasseln am Ende des Inspiriums ist noch hier und da zu hören, in seiner Localisation und Intensität aber stark wechselnd. Die Herntöne sind leise, aber rein. Der Herzspitzenstoss ist an normaler Stelle innerhalb der Mamillarlinie fühlbar. Die absolute Herzdämpfung ist nicht oder kaum noch vergrössert, die Breite der relativen Herzdämpfung beträgt im $1 V$. Intercostalraum $14 \mathrm{~cm}$. An den Abdowinalorganen ist nichts Anormales nachzuweisen, der Harn war in den letzten 8 Wochen eiweissfrei. Die subjectiven Beschwerden bestehen noch in Schmerzen in beiden Brustseiten, besonders beim Gehen und Sitzen, sowie über Druck in der Magengegend. Die Schmerzen in der Her'gegend, das Herzklopfen, die Kurzathmigkeit und der Hustenreiz seien zwar nicht ganz behoben, aber wesentlich gebessert.

In Juni 07 machte der Patient eine Badecur in Soden durch, welche aber keine wesentliche Aenderung des Befundes herbeiführte. Der Zustand war in der Folgezeit ein ziemlich gleichmässiger, aber der chronische Katarrh der Luftwege zeigte im Allgemeinen eine Neigung zu fortschreitender Verschlimmerung durch Vebergang auf die feineren Luftwege. Ein Gutachten vom 28. 3. 08 macht folgende Angaben: Er klagt noch über allgemeine Kraftlosigkeit und Mattigkeit, unruhigen Schlaf, Schmerzen in der Herzgegend, Husten und Auswurf, nur die Kurzathmigkeit sei etwas besser geworden. Dabei sieht Patient ziemlich wohl aus, es zeigt sich aber eine auffallende Abmagerung. Die Musculatur ist schlaffer als früher, an Körpergewicht hat er etwa 
$5 \mathrm{~kg}$ im Laufe eines Jahres verloren. Der Puls ist kräftig, aber leicht unregelmässig, zählt etwa 100 Schläge in der Minute. Die Herzdämpfung ist nicht vergrössert, die Herztöne sind etwas leise, aber rein, Lungengrenzen normal und gut verschieblich, keine abnorme Dämpfung bei der Percussion nachweisbar. Das Athemgeräusch ist vesiculär über beiden Spitzen, hinten etwas rauh und begleitet von zahlreichen, meist trockenen kleinblasigen Geräuschen während des Inspiriums besonders über beiden Unterlappen und der linken Lungenspitze, aber auch über den anderen Theilen der Jungen. Abdominalorgane o. B., insbesondere Leber nicht vergrössert, Harn eiweissfrei.

Auf Grund dieses Befundes machte Patient eine zweite Badecur in Soden von Mitte Mai bis Mitte Juni 08 durch and diese hatte eine wesentliche Besserung zur Folge, Husten und Auswurf bestehen noch ein wenig, keine Herzbeschwerden, dagegen war die Kurzathmigkeit nicht ganz behoben. Der Puls war kräftig, regelmässig und kaum beschleunigt, die Herztöne rein bis auf eine Veränderung des A themgeräusches hinten unten beiderseits, wo es etwas scharf und rauh ist.

In der folgenden Zeit war der Mann dauernd arbeitsfähig, die einzigen persistirenden Erscheinungen bestehen in einer gewissen nervösen Schwäche und Disposition zu Bronchitiden.

Ausser diesen drei Fällen sind mir noch die beiden folgenden bekannt geworden: Ein Aufseher erlitt im Januar 1905 bei einer Störung an der Apparatur, welche zur Darstellung des Phosgens aus CO und $\mathrm{Cl}_{2}$ benutzt wird, indem ein Abflussrohr eines Bebälters, in welchem sich das bei der Phosgenreinigung gebildete Antimonchlorid ansammelt, undicht wurde, eine Vergiftung. Es entstand cin acutes Lungenoedem mit schaumigem und zähem Auswurf. Der Mann wurde mehrere Tage mit fortgesetzten Sauerstoffinhalationen behandelt, dann nahm er die Arbeit wieder auf. Der Mann ist seither dauernd arbeitsfähig, doch giebt er an, seit jener Zeit gegen Phosgen sehr empfindlich geworden zu sein. Ausführlichere schriftliche Aufzeichnungen über den Fall liegen meines Wissens nicht vor.

Der zweite weitere Fall betrifft einen Arbeiter eines Farbstoffbetriebes. Der Mann hatte angeblich am 17. 4.05 Phosgen eingeathmet, arbeitete aber noch bis zum Ende der Arbeitszeit, 6 Uhr Abends. In der Nacht trat ein acutes Lungenoedem ein, und der gegen Morgen herbeigerufene Arzt fand die Situation hoffnungslos, der Mann starb am 18. 4. um 9 Uhr früh. Der Fall wurde obducirt, das Obductionsprotokoll hat mir nicht vorgelegen. Weitere Fälle waren trotz sorgfältiger Nachforschungen nicht zu ermitteln.

Die im Vorstehenden genauer berichteten drei Fälle sind mit denen identisch, welche einer Publication von Herrn Prof. Rumpf zu Grunde liegen ${ }^{1}$ ); wenn ich mir einige Bemerkungen zu dessen Arbeit gestatten möehte, so glaubo ich dies dadurch zu rechtfertigen, dass mir sowohl die Fälle wie die allgemeinen, dabei in Betracht kommenden Verhältnisse genau bekannt sind.

Die Vorgeschichte der drei Fälle ergiebt, dass die Erkrankungen nicht nur durch $\mathrm{POCl}_{3}$ hervorgerufen wurden, sondern dass auch dem

1) Th. Rumpf, Ueber Vergiftung durch Phosphoroxychlorid. Med. Klinik. Jahrg. IV. No. 36. 6.9. 08 . 
Phosgen dabci eine wesentliche Bedeutung zukam, dass ferner die drei Patienten schon vorher der Einwirkung der beiden Gifte, insbesondere aber des Phosgens, schon des Oefteren ausgesetzt waren, wenn auch nicht in dem Maasse, dass einer derselben schon ernstlich unter der lntoxication golitten hätte. Wenn dies in den Ausführungen jener Publication nicht zu Tage tritt, so liegt dies daran, dass das betreffende Actenmaterial nur die Vergiftung mit $\mathrm{POCl}_{3}$ erwähnt und als einmaligo Betriebsunfälie in den Vordergrund stellt, es ist aber nach dem thatsächlichen Sachverhalt keineswegs angängig, diese drei Fälle als beruhend auf einer einmaligen Inhalation von $\mathrm{POCl}_{3}$ darzustellen.

Was nun einen der interessantesten Punkte, die Betheiligung des Herzens, anbetrifft, so bemerkt Rumpf: „Die Dilatation des Herzens in Folge Chlorgaseinathmung ist demgegenüber eine kaum beschriebene Erscheinung. Allerdings betont Harnack in seiner Arbeit über Vergiftungen in dem Handbuch von Ebstein und Schwalbe S. 547, dass im ätiologischen Zusammenhang mit Chlorvergiftungen auch das Vorkommen von Herzlähmungen nicht undenkbar sei. Bei anderen Autoren, insbesondere v. Jaksch in Nothnagel's Handbuch der speciellen Pathologie, konnte ich klinische Belege für eine Betheiligung des Herzens nicht finden. Demgemäss kann eine Dilatation des Herzens nicht wohl dem regelmässigen Symptomenbilde der Chlorvergiftung zugerechnet werden." Demgegenüber darf darauf hingewiesen werden, dass zwar eine specifische Betheiligung des Herzens an der Vergiftung mit Chlor oder gasförmiger $\mathrm{HCl}$ meist nicht auftritt, schon ans dem Grunde, weil die localen Wirkungen auf den Respirationstractus so im Vordergrunde stehen, dass entweder eine energische Reaction des Individuums erfolgt, so dass keine grösseren Mengen resorbirt werden können, oder das Individuum geht eben an diesen localen Wirkungen rasch 20 Grunde; dagegen ist die Betheiligung des Herzens sehr bekannt bei einem viel benutzten chlorhaltigen Körper, nümlich dem Chloroform. Berücksichtigt man, dass nicht das $\mathrm{POCl}_{3}$, sondern das $\mathrm{COCl}_{2}$ die Hauptrolle bei den vorliegenden Vergiftungen spielt, so wird das Krankheitsbild verständlich, da über dio Wirkung des Chloroforms und seiner Zersetzungsproducte eine Fülle von Jiteraturangaben vorhanden sind, aus denen hervorgeht, dass diese Substanzen zu Verfettungen des Myocards führen. Diese Befunde sind sowohl pathologisch-anatomisch wie klinisch genau durchgearbeitet und durch die histologisch nachweisbare Fettdegeneration der erwähnten Organe werden die Krankheitsbilder in ihrem klinischen Verlaufe völlig: erklärt, was aber an diesen Krankengeschichten werthvoll und interessant ist, ist, dass diese fettigen Degenerationen unter Umständen mehr minder vollständig in längerer Zeit ausheilen künnen. Das ist bis jetzt noch nicht in dieser Schärfe betont worden, wenn es auch in den Erörterungen über die Nachwirkung der Chloroformnarkose oft gestreift wurde.

Rumpf ist geneigt, in der Vergiftung mit $\mathrm{POCl}_{3}$ eine Combination der Giftwirkung des $\mathrm{P}$ mit der des Chlors zu erblicken, betont aber selbst die Verschiedenheit der vorliegenden Fälle von Phosphorvergiftungen, namentlich das Fehlen des Icterus. Umgekehrt tritt aber cin leichter Ieterus als Nachwirkung der Chloroformnarkose häufig auf; also 
selbst wenn dieser vorhanden gewesen wäre, hätte er nicht eine specifische Phosphorwirkung bewiesen, was um so verständlicher ist, wenn man an die pathologische Genese des Icterus denkt, der erst eine secundäre Folge der Leberveränderung ist. Da also die analogen Vergiftungsbilder durch das Chloroform und seine Spaltungsproducte, unter denen das Phosphor von besonderer Wichtigkeit ist, in den Grundzügen die gleichen Erkrankungsbilder, insbesondere fettige Degeneration des Herzens, der Nieren und der Leber hervorrufen können, ist die Annahme einer specifischen Phosphorwirkung in der Vergiftung mit $\mathrm{POCl}_{3}$ keineswegs erwiesen.

Rump betont, dass die Affection der Leber eine directe Folge der Vergiftung sei und nicht etwa als Folge der Herzaffection sich auffassen lasse, dass also keine Staungsleber vorliege, wie aus dem Fehlen von Oedemen und Ascites hervorgehe, das ist sicher zutreffend. Da indessen in allen drei Fällen nicht nur eine Schädigung des Herzens, sondern auch eine wesentliche Belastung des kleinen Kreislaufes vorlag und namentlich in dem III. Falle (dem zweiten der Rumpf'schen Publication) eine Cyanose wiederholt festgestellt wurde, so ist das Moment der Staunng doch nicht ganz zu eliminiren.

Es ist stets $z u$ betonen, dass in den vorliegenden Fällen der klinischen Beobachtung kein reines und eindeutiges Material vorlag, dass diese daher auch zur Fundirung bindender Conelusionen weniger geeignet erscheinen, andererseits sind diese klinischen Beobachtungen doch von grösster Wichtigkeit, denn ihre praktischen Interessen sind eigentlich die maassgebenden.

Die Einwirkung des Phosgens auf den Menschen ist nun ausser den Intoxicationsfällen, wie sie in der chemischen Industrie vorkommen können, noch von einer anderen Seite her wichtig. Es ist bekannt, dass das Chloroform, wenn es bei künstlicher Beleuchtung, Petroleum- oder Gaslicht, benutzt wird, sich zersetzen kann, und dass die entstehenden Zersetzungsproducte eine sehr schädliche Wirkung auf die untersuchten Personen haben. Wenn man in einem mit Gaslicht beleuchteten Saale operirt, so kann es, wie Stobwasser ${ }^{1}$ ) ausführte, oft vorkommen, dass Operateur und Assistent nach Verlauf von einer balben Stunde von einem heftigen Hustenreiz befallen werden, der erst mit dem Verlassen des Raumes verschwindet. Stobwasser behauptet, dass die Zersetzungsproducte des Chloroforms $\mathrm{CO}, \mathrm{Cl}$ und $\mathrm{HCl}$ seien. Er machte Versuche, indem er Meerschweinchen in einem Raume hielt, in dem eine Gasflamme brennte und Chloroform zuströmte. Die Thiere gingen zu Grunde und bei der Autopsic fanden sich Veränderungen der Lunge, bronchopneumonische Herde, Lungenödem und Hämorrhagien.

Auch Richet ${ }^{2}$ ) erwähnt, dass unter den angeführten Bedingungen operirte Personen zuweilen unter den Erscheinungen plötzlich einsetzender Asphyxie zu Grunde gehen. Bréaudat³) untersuchte die Zersetzungsproducte des Chloroforms durch Gaslicht und ferner verschiedene Halo-

1) Stobwasser, Berliner klin. Wochenschr. No. 8-10 u. 34. 1889.

2) Richet, Dictionaire de physiologie. article Chloroforme. T. III. p. 634. 1898.

3) Breaudat, De la chloroformisation à la lumière du gaz. Paris 1892. 
genkohlenwasserstoffe, aber kein Phosgen. Dagegen behauptete Dastre ${ }^{1}$, dass das wesentlichste Zersetzungsproduct das Phosgen sei und schreibt ihm als Wirkung einen langdauernden Hustenreiz und die Entstehung katarrhalischer Pneumonien zu. Die Wirkung der Zersetzungsproducte wird in gleicher Weiso von Wilson ${ }^{2}$ ) und Marquart ${ }^{3}$ ) angegeben. Wilson eitirt einen Fall, wo der Operirte und der $\Lambda$ rzt zu Grunde gingen, während zwei Assistenten längere Zeit krank waren.

Betagh ${ }^{4}$ ) beschrieb zwei Fälle von derartigen Intoxicationen. Im ersten Falle hatte die Operirte - Laparotomie bei einer 23 jührigen Frau wegen Orarialkystom — nach dem Erwachen zunächst unstilibares Erbrechen, Collapsanfälle und convulsivische Zuckungen mit kleinem und beschleunigtem Puls. Im Harn trat Eiweiss auf und am folgenden Tage Hämoglobin und Cylinder. Dieser Zustand, der von einem leichten Icterus begleitet war, besserte sich nach einigen Tagen, immerhin blicb cine leichte Nephritis zurück mit geringer Nlbuminurie und granulirten Cylindern. In dem anderen Falle trat nach einer Radicaloperation einer Inguinalhernie zwölf Stunden nach der Operation Tod im Collaps ein. Betagh führte daraufhin eine Anzahl von Versuchen aus, indem er Thiere - Hunde und Kaninchen - in der Nähe einer Gasflamme narkotisirte oder das Gemisch von Chloroform und seinen Zersetzungsproducten, das in der Umgebung einer Gasflamme entstanden war, unter die Maske leitete. Ein Kaninchen starb nach einer derartigen Narkose nach $3 / 4$ Stunden. Eine halbstündige Narkose reichte zur Herbeiführung des Todes nicht aus, aber die Thiere zeigten die Erscheinungen einer Nephritis, Albuminurie, manchmal mit Hämaturie und Cylindern im Harn. Bei den meisten sank der Hämoglobingehalt des Blutes beträchtlich. Bei den zu Grunde gegangenen Thieren fanden sich in erster Linie Veränderungen der Nieren, Desquamation des Glomerulusepithels, intratubuäre Hämorrhagien und Degeneration des Tubuliepithels, ferner fettige Degeneration der Leber, des Myokards und der Nieren. Die spectroskopische Blutuntersuchung ergab das Hämoglobinspectrum und völliges Fehlen des Kohlenoxydhämoglobins.

Maurange ${ }^{5}$ ) beschreibt die Symptome, die er an sich selbst und seiner Assistenz bcobachtet hatte. Er erwähnt eine heftige Reizung der Conjunctiven, der Nasen- und Rachenschleimhaut, dann einen trockenen, heftigen Husten, der bei jeder Inspiration zunimmt und zu einer mehr oder minder heftigen Dyspnoe führt. Joubert ${ }^{6}$ ) erinnert an die Zersetzbarkeit des Chloroforms an der Luft und im Sonnenlicht und betont die

1) Dastre, Les anesthésiques. Physiologie etapplication chirurgicale. Paris 1890.

2) Wilson, The lancet, 24. 6. 1899. Citirt bei Armand und Bertier in Revue de cbirurgie. 1905. p. 32.

3) Marquart, 'Traité de thérapentique. Article chloroforme. T. I. p. 295. $3 e$ édition 1898.

4) Betagh, Il policlinico, 1905 .

5) Maurange, Accidents de la chloroformisation. Gazette hebdomadaire. No. 105. 1899.

6) Joubert et Domergue, Note sur la décomposition des vapeurs de chloroforme. Gazette des Hòpitaux. p. 737. 1905. 
Verschiedenartigkeit der Zersetzungsvorgängo je nach den äusseren Umständen. Armand und Bertier ${ }^{1}$ ) untersuchten die Zersetzungsproducte des Chloroforms speciell mit Hinsicht auf das Phosgen. Es gelang ihnen nicht, Kohlenoxyd im Blute von mit Phosgen vergifteten Thieren nachzuweisen; sie fanden bej der spectroskopischen Untersuchung das typische Oxyhämoglobinspectrum, das bei Zusatz von Schwefelammonium in das Spectrum des reducirten Hämoglobins überging. Delbet ${ }^{2}$ ) wies ebenfalls auf die Gefahren der Chloroformzersetzung hin und suchte namentlich den Einfluss verschiedener Arten von Flammen, Gasflamme, Petroleumflamme, Methyl- und Aethylalkoholfiammen und Paraffinkerzen zu bestimmen. Er hält Petroleumflammen für am günstigsten, doch wird ihm hierin von anderer Seite widersprochen. Dass Phosgen als Zersetzungsproduct des Chloroforms auftrete, wurde von Sehumburg ${ }^{3}$ ) experimentell nachgewiesen, dieser giebt auch an, dass das Blut der Versuchsthiere ein Kohlenoxydhämoglobinspectrum aufwies.

Es wird von verschiedenen Seiten betont, dass die Narkotisirten selbst während der Operation sehr wenig Anormales zeigen. Alles geht gut, bis nach dem Verlauf von $12-24$ Stunden bald für sich allein, bald zusammen mit anderen Symptomen cine icterische Verfärbung der Conjunctiven auftritt und gleichzeitig Eiweiss im Harn erscheint, um in leichteren Fällen einige Tage später zu verschwinden.

Die Zersetzung des Chloroforms bei Gegenwart einer brennenden Gas- oder P'etroleum- oder Spiritusflamme kann in verschiedener Weise verlaufen:

$\mathrm{CHCl}_{3}+\mathrm{O}=\mathrm{COCl}_{2}+\mathrm{HCl}$

$\mathrm{CHCl}_{3}=\mathrm{C}+\mathrm{HCl}+\mathrm{Cl}_{2}$

$\mathrm{CHCl}_{3}+\mathrm{O}=\mathrm{CO}+\mathrm{HCl}+\mathrm{Cl}_{2} ; 2 \mathrm{CHCl}_{3}+3 \mathrm{O}=2 \mathrm{COCl}_{2}+\mathrm{H}_{2} \mathrm{O}+\mathrm{Cl}_{2}$

Es können also in dem entstehenden Gemisch von Zersetzungsproducten neben unzersetztem Chloroform $\mathrm{COCl}_{2}, \mathrm{HCl}, \mathrm{CO}$ und $\mathrm{Cl}_{2}$ auftreten, damit sind aber die Möglichkeiten noch keineswegs erschöpft ${ }^{4}$ ).

Während unter diesen Verhältnissen das Phosgen und die anderen Zersetzungsproducte bereits in dem Narkosegemisch enthalten sind, ist auch an die Möglichkeit zu denken, dass eime Spaltung des Chloroforms im Thierkörper stattfinde und hierbei ähnliche Producte entstehen können wie bei der Spaltung des Chloroforms durch eine Gasflamme.

Da nach den geläufigen Anschauungen der Eintritt der Narkose an die Erreichung eines bestimmten Partiärdruckes im Körper des Narkoti-

1) Armand et Bertier, Du danger des vapeurs chloroxycarboniques dans la chloroformisation. Revue de chirurgie, 1905. p. 32.

2) Delbet, Dangers de la chloroformisation en présence des produits de combustion. Archives générales de médecine. p. 2399. Paris 1905.

3) Schumburg, Hygienische Rundschau. 1898. No. 19.

4) Es ist aus Vorstehendem ersichtlich, dass keineswegs immer Phosgen zu entstehen braucht, es hängt dies im Wesentlichen von dem Sauerstoflzutritt ab. Weitere Erörterungen und Literaturangaben finden sich bei II erbringer, Die Demonstration der Zersetzung des Chloroforms im Gaslichte. Archiv f. exper. Pathologie u. Pharmakologie. Bd. 47. 1902. S. 438 und bei Kobert, Lehrbuch der Intoxicationen. Bd. II. S. 888 . 
sirten gebunden ist, so müssen sich die Inhalationsnarcotica bis zu einem gewissen Grade unzersetzt im Körper anhäufen. Das schliesst aber nicht aus, dass doch eine Zersetzung des Narkotikums im Thierkörper stattfinden kann; sehr bedeutend kann diese Zerstörung nicht sein, wie aus der weitgehenden Giltigkeit der physikalisch-chemischen Theorie der Inhalationsnarkose, wie sie von Paul Bert einerseits, von Hans Meyer und Overton andererseits ansgebaut wurde, hervorgeht. Dass aber Inhalationsanästhetica im Thierkörper zersetzt werden können, ist für das Bromäthyl von Dreser ${ }^{1}$ ) nachgewiesen, und diesen Zersetzungen kommt zweifellos eine grosse Bedeutung für dessen Neben- und Nachwirkungen zu. Dreser fand, dass in tödtlich verlaufender Bromäthylvergiftung dieses sich im Körper in andere Bromverbindungen umsetzt, die im Harn nachgewiesen werden können.

Zeller ${ }^{2}$ ) fand im Harn mit Chloroform behandelter Thiere ein entschiedenes Höhergehen des Chlorgehaltes. Eindeutig ist allerdings dieser Befund nicht, denn eine Vermehrung der Chlorausscheidung aus anderen Gründen unter dem Einflusse des Chloroforms ist nicht ausgeschlossen. Ganto ${ }^{3}$ ) fand, dass der Chloroformgehalt eines in der Narkose getödteten Thieres beziehungsweise der seiner Organe sinkt, wenn man dieses Organ eine zeitlang aufbewahrt. Daraus folgt, dass überlebendes und todtes Gewebe die Fähigkeit besitzt, Chloroform zu zerstören, und dies wurde von Schneider ${ }^{4}$ ) quantitativ verfolgt, welcher fand, dass Organbrei von Muskeln, Leber und $\mathrm{Milz} 10 \mathrm{pCt}$. und mehr des zugesetzten Chloroforms zerstören konnte.

Welche Zersetzungsproducte aus dem Chloroform auf diese Weise im Thierkörper entstehen können, lässt sich natürlich nur vermuthungsweise sagen, aber es ist wahrscheinlich, dass gerade sie für die Nachwirkungen des Chloroforms verantwortlich gemacht werden müssen, da diese zu einer Zeit auftreten, wo bereits der grösste Theil des Narkotikums unzersetyt durch die Athemluft wieder ausgeschieden wurde. Da neben diesen verwickelten Verbältnissen noch die Reactionsfähigkeit des Organismus in Betracht kommt, die Widerstandsfähigkeit der Nieren, der Lungen, des Myocards, so wird es verständlich, dass die postnarkotische Wirkung des Chloroforms innerhalb relativ weiter Grenzen variiren kann.

Weder dic klinischen Erfahrungen am Menschen über Phosgenvergiftungen in der 'Technik, noch die Folgeerscheinungen der Intoxication mit mehr minder weit zersetzten Chloroformnarkosegemischen reichen zu einer völligen Kenntniss der Phosgenvergiftung aus; ihnen kommt zwar zunächst die grösste praktische Bedeutung für den Hygieniker, den Internisten und den Chirurgen zu, es bedarf aber weiterhin des Thicr-

1) Dreser, Zur Pharmakologie des Bromäthyls. Arch. f. experiment. Pathol. u. Pharmakol. Bd. 36. S. 285.

2) Zeller, Ueber die Schicksale des Chloroforms im Organismus. Zeitschr. f. physiol. Chemie. Bd. VIII. S. 70.

3) Gantow, Der Chloroformgehalt der Organo während der Narkose. Inaug.Dissert. Giessen. 1905.

4) Karl Schneider, Die Zersetzung des Chloroforms durch thierische Gowebo. Inaag.-Diss. Giessen 1905. 
versuches, um experimentell die toxische Wirkung des Phosgens quantitativ zu bestimmen und in ihren einzolnen Folgen isolirt zu untersuchen.

Die zunächst im folgenden beschriebenen Versuche wurden in der Weise ausgeführt, dass das Phosgen auf dem Wege des Respirationstractus den Thieren zugeführt wurde. Dabei wurden vorwiegend Ratten benutzt. Dazu wurde eine Lösung von Phosgen in Benzol benutzt. Durch besondere Controlversuche wurde festgestellt, dass die dabei angewandten Benzolmengen toxische Effecte gaben, welche sich von denen des Phosgens scharf trennen liessen und beträchtlich gegen die Phosgenwirkung zurücktraten.

Der Gehalt der mir zur Verfügung stehenden Phosgenbenzollösung liess sich auf zwoierlei Weise, die sich gegenseitig controliren, bestimmen, einmal rechnerisch aus den specifisehen Gewichten, zweitens analytisch.

Das specifische Gewicht der Phosgenlösung betrug bis $18,2^{\circ} 0,963$. Das specifische Gewicht des reinen flüssigen l'hosgens bei $0^{0}$ beträgt 1,432, das speeifische Gewicht des Benzols bei $20^{\circ} 0,879$. Daraus ergiebt sich für dic Phosgenmenge $\mathrm{x}$ in einem Cubikcentimeter folgender Ansatz $=$

$$
\begin{gathered}
(1-x) \cdot 0,879+x \cdot 1,432=0,963 \\
0,879+x(1,432-0,879)=0,963 \\
x=0,152 \text { in } 1 \text { ccm. }
\end{gathered}
$$

also betrug der Phosgengehalt der Lösung $15,2 \mathrm{pCt}$.

Zweitens lässt sich die Phosgenmenge analytisch direct bestimmen, indem man den Chlorgehalt der Lösung feststellt. Zu diesem Zwecke werden $5 \mathrm{ccm}$ Phosgenlösung abpipettirt und in einem Literkolben mit festem Baryumhydroxyd und Wasser auf dem Wasserbad längere Zeit erwärmt, um das Phosgen zu zerstören. Es wird von dem überschüssigen Baryumhydroxyd und dem entstandenen Baryumcarbonat abfiltrirt, der Rüskstand ausgewaschen und das Filtrat auf $500 \mathrm{cem}$ aufgefüllt. Ein aliquoter Theil des Filtrats wurde nach Volhard titrirt und es ergab sich der Phosgengehalt der Lösung zu 15,57 pCt.

Beide Werthe stimmen ganz gut überein, doch ist der analytisch bestimmte der genauere; der Unterschied rührt daher, dass die specifischen Gewichte, welche zu Grunde gelegt wurden, nicht alle auf dieselbe Temperatur $\left(18,2^{\circ}\right)$ bezogen sind. Immerhin ist aber gerade das rechnerische Verfahren das elegantere und bequemere.

Aus dem Procentgehalt der Mischung lässt sich num berechnen, dass die Verdampfung von einem Cubikcentimeter der Mischung, wenn der Dampf den Bedingungen des Gaszustandes folgt, in 10 Liter Luft ein Athemgemisch liefert, das 2,21 Volumprocent Benzol and 0,494 Volumprocent Phosgen enthält ${ }^{1}$ ). Zur Herstellung des Athemgemisches diente der von Dreser construirte Apparat'). An diesem hatte aber der Ge-

1) Die Grundlagen der Rechnung sind ausführlich behandelt bei W. Hennicke, Vergleichende Untersuchungen über die Gefährlichkeit der gebrüuchlichen Inbalationsanästhetika. Inang.-Diss. (Bonn.) Berlin 1895. S. 17-22.

2) Dreser, Die Dosirung der Inhalationsanästhetika. Arch. 1. experiment. Pathol. u. Pharmakol. Bd. XXXVII. 1896. S. 375. 
brauch im Laboratorium zu einer recht geschickten Modification Anlass gegeben; Dresor schreibt; „Die Excursion, welche der Spirometercylinder bei seinem Auf- und Niedergange macht, blcibt ein für allemal dieselbe, nämlich 10 Litern Luft entsprechend. Um versehieden stark dosirte Gcmische herzustellen, muss aber die Höhe der austreibenden $\mathrm{Hg}$-Säule veränderlich sein, was dadurch ermöglieht wird, dass die an dem Spirometercylinder befindliche Schnur an einem Storehschnabel angreift. Der unterste Drehpunkt des, Storchschnabelviereckes' ist fixirt, macht also gar keine Excursion; würde man an dieser Stelle des Storchschnabels das Niveaugefäss anhängen, so wird gar kein Anästheticum in den Luftcanal entlecrt. Je höher man das Niveaugefüss an den Storchschnabel anhängt, um so höher wird die austreibende Hg-Säule, die stets mit derselben Geschwindigkeit sich aufwärts bewegt, mit der sich der Cylinder senkt." Diese Einrichtung wurde später ersetzt durch eine Kurbeleinrichtung, welche ebenso wie ein Storchschnabel functionirt, abcr viel bequemer ist. An der Kurbelachse ist rechtwinklig zu ihr ein Metallschenkel befestigt, auf welchem cin zweiter Schenkel verschieblich feststellbar ist. Dieser zweite Schenkel trügt an seinem Ansatzstück die Schnur für den Spirometercylinder und auf sich verschiebbar die Aufhängung des Niveaugefässes. Da beide Aufhängungen zu verschiedenen Seiten der Kurbelachse liegen, ist natürlich die Bewegung der an ihnen befestigten Theile gegensinnig. Durch Variation der beiden Hebelarme lassen sich die verschiedensten Coincidenzverhältnisse in der Senkung des Spirometercylinders und Hebung des Niveaugefässes crreichen.

Wie bereits erwähnt ist, wurde eine Lösung von Phosgen in Benzol benutzt, und es muss kur $\%$ auf die Frage eingegangen werden, welcher Einfluss dem Benzol auf dic in den Versuchen beobachteten Erscheinungen zuzuschreiben ist. Dic Versuche wurden bei Zimmertemperatur ausgeführt, der Procentgehalt eines mit Benzol gesättigten Luftgemisches beträgt bei $20^{\circ} 9,95$ Volumprocent, der Benzolgehalt des $\Lambda$ themgemisches beträgt also bei Vergasung von $1 \mathrm{ccm}$ Benzolphosgenlösung etwa cin Viertel des Gehaltes, der bei der Sättigung mit Benzol erreicht wird. Thatsächlich wurden aber viel kleinere Quantitäten, von $1 / 2 \mathrm{ccm}$ abwärts, benutzt, so dass etwa mit $1 / 8$ der Sättigung mit Benzol und weniger gearbeitet wurde. Wenn auch bei diesen Concentrationen eine schwere toxische Benzolwirkung nicht $\mathrm{zu}$ erwarten war, so wurden doch entsprechende Controlversuche vorgenommen:

Ratte I. Controlversuch mit $0,84 \mathrm{ccm}$ Benzol $=2,21 \mathrm{Vol} . \mathrm{pCt}$.

28. 11. 06. Anfang 9 Uhr $25 \mathrm{Min}$, viermal 10 Liter Luftgemisch werden durch den Thierrecipienten durchgepumpt. Nach Athmung dieser Luftbenzolmischung liegt das Thier auf den wie gelähmt erscheinenden Hinterpfoten und ist stark unruhig. 5 Min. später liegt das Thier flach auf dem Bauch, dio Athmung ist unregelmässig, zeitweilig treten krampfartige Zuckungen in den Hinterextremitäten auf. Beim Behorchen röchelt das Thier nicht. 9 Uhr 36 Min. die Narkose abgebrochen, hat sich bereits 9 Uhr 40 Min. wesentlich erholt und zeigt in der Folge keine abnormen Erscheinungen.

Ratte II. Benzolcontrolversuch. 0,42 ccm Benzol = 1,10 Vol.-pCt. Anfang 10 Uhr 1 Min., schnuppert unruhig. 10 Uhr 12 Min. leichtes Schwanken, Athmung stark frequent. Nach $7 \mathrm{mal} 10$ Litern liegt das Thier auf den Hinterbeinen flach, nach 
8mal 10 Litern starke klonische Zuckungen der Rumpfmusculatur. $\Lambda$ thmung dyspnoisch. 10 Uhr 16 Min.: die Narkose ist ruhiger, aber die Athmung immer noch stark frequent. Ende 10 Uhr 17 Min., das Thier erholte sich rasch und zeigte in der Folge keine krankhaften Eirscheinungen.

Beide Thiere gingen nach 7 und 8 Wochen zu Grunde. B $\theta$ i der Inspection des ersten Thieres sind die Lungen collabirt, lufthaltig, von hellröthlicher Farbe, die Pleuren spiegelnd. Beide Ventrikel des Herzens sind systolisch contrahirt, die Vorhöfe stark mit Blut gefüllt, Leber etwas hyperämisch und etwas vergrössert. Dünndarmschlingen lebhaft injicirt, Darmschleimhaut glasig geschwollen. Bei der Inspection der anderen Ratte finden sich leichte Hypostasen in den ventralen Zipfeln des rechten Mittel- und Unterlappens und dem Apex des rechten Oberlappens, Lungen sonst normal. Herz und Leber ohne Besonderheiten. Die Dünndarmschleimhaut goröthet und stark injicirt, stellenweise mit leichten Hämorrhagien. Daraus wurde gefolgert, dass die Thiere nicht an einer Benzolwirkung, sondern an einem intercurrenten Darmkatarrh zu Grunde gegangen sind.

Nach diesen Vorversuchen konnte mit den Phosgeninhalationsversuchen begonnen werden.

Ratte III. 0,2 Volum-pCt. Phosgen. Anfang 10 Uhr 38 Min. Die Ratte zeigt leichte Unruhe, speichelt stark und sitzt mit gekrümmtem Rücken da. Zuweilen schüttelt sie den Kopf, wie wonn sie etwas abschütteln wolle. Die Augen thränen stark, die Athmung ist kaum beschleunigt, zeitweise schnappend. 10 Uhr 45 Min.: Kann sich kaum aufrecht halten. Das Bild differirt wesentlich von der Benzolinhalation, indem die Athmung dauernd verlangsamt ist und dyspnoisch bleibt. Ende 10 Uhr 52 Min. Tod 11 Uhr 59 Min. Sie hatte sich vorher ganz gut erholt, nur war die Athmung dauernd dyspnoisch geblieben. Das Thier zeigte starke Unruhe und war noch wenige Minuten vorher auf dem T'isch umhergelaufen. Plötzlicher Collaps 11 Uhr 50 Min., stirbt mit einzelnen dyspnoischen Athemzügen.

Die sofort vorgenommene Inspection ergiebt ein interessantes Bild: Die Lungen sind ad maximum gebläht; der rechte Mittellappen von rechts her, der linke Unterlappen von links her verdecken in situ das Herz beinahe völlig. Die Lungen collabiren nicht und fühlen sich elastisch an. Unter der Lungenpleura, namentlich an den Rändern der Lappen, finden sich deutlich erkennbare Luftbläschen, welche durch die Pleura durchschimmern. Stellenweise, weiter vom hand entfernt, bilden sie sternförmige Figürchen. Die Zeichnung der Lungenläppchen tritt scharf hervor. Bei einem Einschnitt erweist sich die Lunge als blutarm, dagegen entleert sich eine seröse Flüssigkeit (Lungenödem). Herz systolisch contrahirt, Vorhöfe mässig mit Blut gefüllt, sonst ohne Besonderheiten.

Es handelte sich also um ein interstitielles Lungenemphysem, das man sich so entstanden denken kann, dass die durch die Zersetzung des Phosgens entstehende IICl in statu nascendi die Alveolarwandungen durchsetzt hat und die Luft bei der Exspiration ins perialveoläre Bindegewebe trat und sich unter der Pleura ansammelte, ein Vorgang, der unterstütat wurde durch den reflectorischen Glottisverschluss. Fs ist verständich, dass diese Luft bei den folgenden Exspirationen nicht ausgetrieben, sondern nur comprimirt und im interstitiellen Lungengewobe weitergepresst wurde und so einerseits in toto mehr oder weniger als schädlicher Raum wirkte, andererseits die Gewebsläsion verwehrte. '/usammen mit der Exsudation der serösen Flüssigkeit in die Alveolen ist damit eine Erklärung der Dyspnoe, an welcher das Thier rapide zu Grunde ging, gegeben.

Die spectroskopische Blutuntersuchung ergab ein Oxyhämoglobinspectrum. Die Brustorgane wurden im Ganzen in Sublimat fixirt, am nächsten Tage zertheilt, mit Jodalkohol behandelt und in Paraffin eingebettet.

Ratte IV. 29. 11. 06. Anfang $10 \mathrm{Uhr} 15 \mathrm{Min}$. 0,05 Volum-pCt. Phosgen. Um 10 Uhr 24 Min. hat das Thier die Augen halb zugeknillen, schnuppert lobhaft. 
10 Uhr 27 Min. Athmung sehr llach, schüttelt zeitweilig mit dem Kopf, zcitweilig Zuckungen in den Hinterbeinen. Es werden achtmal je 10 liter Luftgemisch durchgepumpt, Ende 10 Uhr $30 \mathrm{Min}$.

Das Thier erholt sich etwas, doch bleibt die $\Lambda$ thmung dysproisch und die cinzelnen Athemzüge sind ungleich tief; im Laufe des Mittags wird das Thier sohr unruhig und klettert aus seinem Behälter, einem Drahtkorb, heraus und läuft auf dem 'Tisch umber. Gegen 2 Uhr 30 Minuten werden die Athemzüge stark vertieft, dyspnoisch, es stellt sich ein Collaps ein und das Thier stirbt relativ plötzlich um 2 Uhr $50 \mathrm{Min}$.

Die sofort vorgenommene Inspection liefert ein von dem vorigen Falle äusserlich wesentlich abweichendes Bild: die Lungen sind etwas retrahirt, fühlen sich derb an und sind seht blutreich, namentlich die Oberlappen sind dunkelbraunrot, ebenso die abschüssigen Partien des rechten Mittel- und Cnterlappens. Dabei besteht an den Rändern ein interstitielles streifenförmiges Emphysem, und ein ebensolches ist subpleural an anderen von den Rändern entfernteren Stellen in Form von Herden vorhanden. Diese emphysematischen Stellen erscheinen bräunlich-gelblich, sodass die Junge äusserlich in Fürbung und Aussehen Aehnlichleit hat mit einer Lunge mit grossen peribronchitischen Tuberkelherden.

Von den Brustorganen wurde eine farbige Zeichnung hergestellt, dann wurden sie als Ganzes in Sublimat fixirt und nach entsprechender Vorbehandlung in Paraffin cingebettet.

Ratte V. 29.11.06. Das Thier wurde um $10 \mathrm{Uhr} 34 \mathrm{Min}$. in den Recipienten verbracht und es wurden dreimal je 10 Liter Phosgenluftgemenge von $0,05 \mathrm{Volum}-\mathrm{pCt}$. Phosgen durchgepumpt. Dauer des Versuches 5 Min. Das Thier zeigt Unruhe und Erschwerung der Athmung, die Augen werden fest geschlossen. Nach Beendigung des Versuches erholt es sich etwas, zeigt später starke motorische Unruhe, die Athmung bleibt dyspnoisch. Der Tod tritt, wie in den vorhergehenden Fällen, ziemlich plötzlich um $1 / 44$ Uhr ein.

Die Inspection ergiebt: Lungen etwas retrahirt, von derbelastischer Consistenz, Oberlappen und abschüssige Lungenpartien dunkelbraunroth, fleckenweise subpleurales interstitielles Emphysem und interstitielles Randemphysem. In der Plourahöhle findet sich freie seröse Flüssigkeit, ebenso im Herzbeutel. Die Ventrikel sind systolisch contrahirt, beide Vorhöfe sind stark mit Blut gefüllt.

Aus dem Vorhergehenden folgt, dass bei einer Concentration von 0,05-0,2 Volum-pCt. Phosgen in der Athemluft die Thiere unter schweren J äsionen der Tauge innerhalb weniger Stunden zu Grunde gehen; daher wurde die Phosgenlösung soweit verdünnt, dass $0,5 \mathrm{ccm}$ einem Gehalt des Luftgemenges von 0,0247 Volum-pCt. Phosgen entsprachen.

Ratte VI am 30.11.06 um 10 Uhr 15 Min. eingesetzt; das Thier ist in den ersten 3 Minuten ruhig, wird dann unruhig, kneift die Augen zu und schnuppert lebhaft. Die $\Lambda$ thmung ist erschwert und unregelmässig. 10 Uhr 19 Min.: Die Ratte schwankt und sinkt um (Benzolnarkose). 10 Uhr 29 Min.: Die Athmung ist stark beschleunigt, zeitweiliges Zucken in den Hinterbeinen. Ende 10 Uhr 35 Min. Das Thier erholt sich gut, schläft am Mittag und ist um $1 / 2^{4}$ Uhr gut im Stande, doch ist die Athmung dyspnoiseh.

Ratte VII. Dieselbe Phosgenconcentration. Beginn 11 Uhr 30 Min. Das Thier richtet sich im Recipienten auf, ist unruhig, sucht an den Wänden emporzusteigen. 11 Uhr 34 Min.: Läuft unter dem Recipienten einher, taumelt etwas. Dauer des Versuches 5 Minuten. Mittags $1 / 24$ Uhr ganz gut im Stande, die Athmung ist nicht auffallend dyspnoisch. 
Im Jaufe der Nacht starben boide Thiere. Die Inspection ergiebt bei beiden Thieren den gleichen Befund: Die Musculatur ist beim Durchschneiden feucht, Nieren und Jeber blutreich, aber sonst ohne Besonderheiten. Die Lungen sind retrahirt, es findet sich in der Pleuraböhle ein Erguss von mehreren Cubikcentimetern klarer Flüssigkeit. Die lungen sind, namentlich in den abschiissigen Partien, stark hyperämisch, es findet sich ein fleckiges und streifiges interstitielles Emphysem.

Ratte VIII. 3. 12. 06. 0,0123 Volum-pCt. Phosgen. Anfang 10 Uhr 39 Min. Das Thier richtet sich an der Wand des Recipienten auf, ist unruhig, schnuppert lebhaft und schüttelt sich. $10 \mathrm{Uhr} 42 \mathrm{Min}$.: Taumelt stark, ist unruhig und kann sich laum auf den Hinterbeinen halten. 10 Uhr 43 Min.: Liegt auf dem Riucken, kilonische '/uckungen in den Hinterextremitäten. Bleibt mit leichten klonischen Zuckungen bis zum Ende des Versuches liegen. Dauer 15 Minuten.

Ratte IX. Das Gemisch wurde in diesem und den beiden folgenden Versuchen (Ralte $\mathrm{X}$ und $\mathrm{XI}$ ) beibehalten. Anfang $10 \mathrm{Uhr} 55$ Min. Das Thier ist von Anfang an unruhig, sitzt dann mit stark gekrümmtem Rücken da. Gegen Ende des Versuches taumelt es und kann sich kaum noch auf den Beinen halten. Ende 11 Uhr 1 Min.

Beide Thiere leben noch am 1.2.07, wo ihre Controle abgebrochen wurde, und zeigen äusserlich normales Verhalten.

Ratte X. 7. 12.06 um $1 \mathrm{Uhr} 57$ Min. eingesetzt. Dauer des Versuches bis 2 Uhr 12 Min. Leichte Unruhe, Athmung stark beschleunigt. Sitzt gegen Ende des Versuches zusammengekauert und mit den Hinterbeinen zitternd da, erbolt sich in den nächsten Stunden gut und zeigt in den folgenden 'lagen normales Verhalten. Am 15. 12. wurde sie wieder für 15 Minuten in den Recipienten gebracht, starke Benzolnarkose, ist nach dem Versuche recht angegrillen. erbolt sich aber rasch und ist am folgenden Tage äusserlich normal. $\Lambda \mathrm{m}$ 2. 1. 07 wurde das Thier wiederum von 11 Uhr 48 Min. bis 12 Uhr 3 Min. der Phosgeninhalation ausgesetzt. Es wurde am 17.1.07 zusammen mit dem folgenden 'l'hiere getïdtet.

Ratte XI. 7. 12. 06 um 2 Uhr 14 Min, eingesetzt. Dauer 5 Min. Das Thier zeigt die bekannten Erscheinungen von Unruhe und Unbehagen, ist nach dem Vorsuche recht lebhaft, taumelt etwas. Am 13. 12. und am 2. 1.07 wiederum für je 5 Min. der Phosgeneinathmung ausgesetzt, übersteht dieselbe gut und zeigt ausser leichter Dyspnoe normales Verbalten.

Am 17. 1. 07 wurden beide Thiere durch Durchschneiden der Carotiden getödtet. Der Inspectionsbefund der beiden Ratten ist wenig verschieden. Die Nieren sind hyperämisch, die Rindenschicht verbreitert, die Leber ist vergrössert, zeigt deutliche Muskatnusszeichnung. Das Myocard ist blass, trübe. Die Pleuren sind nicht verklebt, glatt, feucht glänzend. Die Lungenpleura zeigt mehrere linsengrosse, pockennarbenähnliche Einziohungen, unter denon frische, verdichtete Bindegewebszüge liegen. Stellenweise finden sich bis linsengrosse, streifige Hypostasen.

Die Brustorgane wurden in Alkohol fixirt, Stückchen von den Nieren, Herz und Leber in Flemming'scher Lösung.

Aus dem Vorhergehenden folgt, dass bei einem Gehalt von $0,02 \mathrm{Vol}$.-pCt. des Inhalationsgemisches an Phosgen die Thiere innerhalb 24 Stunden zu Grunde gehen, während sie bei einem Gehalt von 0,0123 Vol.-pCt. selbst eine mehrmalige Inhalation überstehen und längere Zeit am Leben bleiben können. Die letale Concentration lässt sich also ziemlich scharf bestimmen. Je rascher der Tod eintritt, um so mehr stehen die Veränderungen der Athmungsorgane im Vordergrund, die der anderen Organe brauchen längere Zeit, um sich zu entwickeln.

Die Versuchsserie wurde mit Ausnahme der beiden letzten Versuche $(X$ und $X I)$ im Februar 1907 in genau der gleichen Weise und Reihen- 
folge wiederholt, um /ufülligkeiten auszuschliessen. Die Versuche ergaben aber von Fall zu Fall cine geradezu auffallende Uebereinstimmung mit den hier beschriebenen, so dass diese Versuche als typisch und frei von stark abweichenden individuellen Variationen angesehen werden können, und es überflüssig ist, diese Controlreihe nochmals im Einzelnen zu behandeln. Die festgestellten Werthe dürften in annähernd gleichem Betrage auch für andere Thiorarten und für den Menschen gelten. Es kommt dabei viel weniger auf die absolute Menge Phosgen an, die in den thierischen Körper gelangt und welche festzustellen bei diesen Versuchen fast unmöglich sein dürfte, als auf die Concentration und die Dauer der Einwirkung. Diese Factoren werden aber nicht in gleicher Weise wie die absoluten Dosen von dem Körpergewicht des Versuchsthicres beeinflusst.

Um nun zu einigen Versuchen über dic Phosgenwirkung bei der Einverleibung per os überzugehen, wurde zunächst der Vertheilungscoefficient des l'hosgens zwisehen Benzol und Wasser bei Zimmertemperatur bestimmt, indem $10 \mathrm{cem} 15$ proc. Phosgenbenzollösung mit $50 \mathrm{ccm}$ Wasser im Scheidetrichter geschüttelt und dann getrennt wurden, und in der wässerigen Lösung nach Zerstörung des Phosgens durch überschüssigen Aetzbaryt der Chlorgehalt dureh Titration bestimmt wurde. Der Theilungscoefficient ergab sich zwischen Benzol und Wasser wie 0,764:0,736.

Es wurden nun $50 \mathrm{ccm}$ einer solchen wässerigen l'hosgenlösung, enthaltend 0,736 g Phosgen, einer Katze mittelst Magensonde beigcbracht. Unmittelbar nach der Eingabe ist das Thier etwas unruhig, dann beginnt es stark zu speicheln, vereinzelte Würgbewegungen treten auf, aber kein Erbrechen. Nach 15 Min. war die Athmung sehr frecuent, das $\Lambda$ thmungsgeräusch bei dor Auscultation bei der Inspiration leise und rein, bei der Exspiration rauh. Herzschlag nicht zu lïhlen, Puls der A. brachialis und A. femoralis fadenförmig. Der Allgemeinzustand verschlechtert sich sehr rasch, es tritt mïssige Cyanose auf. Zunge rothviolett. Nach 35 Min. liegt das Thier auf der Seite, die Athmung ist stark dyspnoisch. Das 'Thier stirbt unter leichten Erstickungskrämpfen nach 50 Minuten.

Aus dem angeführten Versuche folgt, dass die Phosgenvergiftung per os mit der Phenolvergiftung das gemeinsam hat, dass kein Erbrechen auftritt.

Bei der Inspection zeigen die Lungen das von den Rattenversuchen her bokannte interstitielle Emphysem an den Randpartien, das übrigens an und für sich nichts Specifisches für die Phosgenvergiftung ist, sondern in Thierversuchen vielfach da beobachtet wird, wo dyspnoische Erscheinungen in der Agonie stärker auftreten. Im Uebrigen sind die Lungen weich, von normaler Färbung und retrahirt. In der Pleurahöhle keine freie Flüssigkeit. Herzmusculatur trübbraunroth, das rechte Herz, Vorhof und Ventrikel schwappend mit tiefbraunrothem Blute gefüllt, das sich beim Schütteln im Reagensglas an der Luft wieder hellroth färbt. Magenschleimhaut in etwa $9 / 3$ ihrer Ausdehnung tief verätzt, mit geronnenem Blutwasser bedeckt; in der Duodenalschleimhaut einige kleine, streifenförmige Blutungen, Dünndarm leer.

Jerz, Theile der Lunge und der Magenwandung werden in Sublimat fixirt und in Paraffin eingebettet.

Das Phosgen entfaltet also bei seiner Einverleibung per os sebr intensive locale Wirkungen als Aetzgilt, was anf dem Froiwerden von Salzsäure beruht, es werden bereits im Magen offenbar erhebliche Mengen resorbirt. Dazu kommt, dass von der relativ sehr beträchtlichen eingeführten Flüssigkeitsmenge ein 'Theil regurgitirt und aspirirt wurde, woraus sich auf die einfachste Weise die Veränderungen der Lungen erklären. 
Es mag hier beiläufig erwähnt werden, dass in derselben Weise wie das Phosgen auch das Phosphoroxychlorid untersucht wurde. In qualitativer Beziehung ergaben sich keine wesentlichen Unterschiede gegen das Phosgen, hier wie dort stehen im Vordergrunde die Reizerscheinungen und Schädigungen der $\Lambda$ thmungsorgane, hier wie dort kommt es zu ausgedehnter fettiger Degeneration der verschiedenen Organe, vor Allem des Myocards, der Nieren und der Leber; es liess sich aber feststellen, dass diese fettigen Degenerationen bei der Vergiftung mit $\mathrm{POCl}_{3}$ noch langwieriger und intensiver waren als bei der Phosgenvergiftung. Da indessen die Einwirkung des $\mathrm{POCl}_{3}$ Anlass zu anderweitigen Erörterungen abgäbe, so soll die vorliegende Arbeit nicht noch damit belastet werden, andererseits sollte es aber im Hinblick auf die klinisch beobachteten Fälle erwähnt werden.

Um nun auf die histopathologischen Erscheinungen bei der Phosgen wirkung überzugehen, liegt es am nächsten, an die bekannten Wirkungen des Chloroforms anzuknüpfen, da dessen Nachwirkungen in ihrem Befunde durchaus in Analogie zur Wirkung des Chloroforms zu bringen sind. 'T'eissier ${ }^{1}$ ) und Patein ${ }^{2}$ ) machten im Jahre 1884 auf die Albuminurie nach Chloroformnarkose aufmerksam, welche sie in rund $78 \mathrm{pCt}$. der Fälle fanden und sie betonen den transitorischen Charakter dieser Albuminurie. Lutze $^{3}$ ) fand in 27 Fällen von Chloroformnarkose 18 mal Albuminurie, deren Dauer meist zwischen 1-4 Tagen schwankte, in einem Falle aber 22 Tage betrug. Weitere Angaben darïber wurden von Luther ${ }^{4}$ ), Rindskopf $f^{5}$ ), Alessandri6), Barbacci und Bebi ${ }^{7}$ ), Wunderlich ${ }^{8}$ ), Friedlaender ${ }^{9}$ ), Niebergall ${ }^{10}$ ), Vidal ${ }^{11}$ ), Le doux ${ }^{12}$ ), Arnozan ${ }^{13}$ ), Inizian ${ }^{14}$ ) und Anderen gemacht. Vergleicht man nun diese verschiedenen Angaben, so fällt auf, wie weit sie hinsichtlich der procentualen Häufigkeit des Auftretens von einander abweichen und es lässt sich dafür weder hinsichtlich

1) 'Teissier vide Stiles and Macdonald, Scottish med. and surg. journal. Jg. VII. 1904. - A ubertin, Les effets tardifs du chloroforme. Thèse deParis. 1906.

2) Patein, De l'albuminurie consécutive aux inhalations chloroformiques. Thèse de Paris. 1888.

3) Lutze, Ueber den Einfluss der Chloroformnarkose auf die menschlichen Nieren. Würzburger Inaug.-Dissert. 1897.

4) Luther, Ueber Chloroformnachwirkung. Münchener med. Wochenschr. 1893. No. 1.

5) Rindskopf, Klinische Beobachtungen über den Einfluss der Chloroformnarkose auf die menschlichen Nieren. Deutsche med. Wochenschr. 1893. No. 40.

6) Alessandri, Degli effetti del chloroformio sull'organismo ed in particolari sui reni. Il policlinico. 1893 .

7) Barbacci e Bebi, Sull'azione dell'etere e del chloroformio sui reni. Il policlinico. 1896.

8) Wunderlich, Klinische Untersuchungen über die Wirkung der Aether» und Chloroformnarkose auf die Nieren. Beitr. zur klin. Chir. 1894.

9) Friedlaender, Ueber die Beeinflussung der Nierenfunction durch die Chloroformnarkose. Vierteljahresschr. f. gerichtl. Med. 1894.

10) Niebergall, Ueber den Einlluss längerer Chloroformnarkose auf Blut und Harn. Inaug.-Dissert. Basel. 1894.

11) Vidal, Influence de l'anesthésie sur les phénomènes cliniques de l'organisme. Thè̀se de Paris. 1887.

12) Ledoux, De l'influence de chloroforme sur les reins. Albuminurie et cylindrurie post-chloroformínues. Thèse de Paris. 1903-1904. 1896.

13) Arnozan, Prognostic des Albuminuries. Congrès de médecine de Nancy.

14) Inizian, De l'intoxication par les dérivés chlorés résultant de la décomposition du Chloroforme. Thèse de Toulouse. 1907. 
der Cualität des Chloroforms noch seiner Quantität oder der Daucr der Narkose eine Beziehung anfstellen, man wird vielmehr dazu gedrängt, oine verschicdene Widerstandsfähigkeit der Nierenepithelien bei den verschiedenen Individuen anzunehmen, wolche theils angeboren, theils durch frühere Schädlichlieiten, Intoxicationen, Infectionen, Gravidität und $\Lambda$ busus von Genussmitteln erworben sein mag. Die Albuminurie verschwindet meist in wenigen 'lagen, sic kann aber auch länger dauern und unter dem Symptomencomplex der Urämie letal endigen. Aehnlich wie bei den Nieren liegen hinsichtlich der Functionsstörung die Verhältnisse bei der lober.

Die morphologische Veränderung, welche der Albuminurie, den Functionsstörungen der Leber und des Herzens zu Grunde liegt, ist im Princip dieselbe, nïmlich eine Cottige Degeneration des Driisenepithels und des Myocards. Auf Grund der zahlireichen vorlicgenden $\Lambda$ rbeiten, erwähnt seien nur Nothnagel ${ }^{1}$ ), Bouchard ${ }^{2}$ ), laborde ${ }^{3}$ ), Toth ${ }^{4}$ ), Ungar ${ }^{5}$ ), Strassmann ${ }^{6}$ ), Ostertag ${ }^{7}$ ), Thiem und Sischer $^{8}$ ),

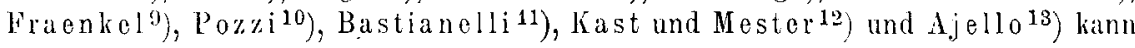
man den Thatbestand dahin zusammenfassen, dass eine Einwirkung auf die Drüsenopitholien und die Muskelfasern des Herzens direct stattfindet und dass daneben eine directe toxische Wirkung auf die rothen Blutkörperchen existirt.

Aehnlich liegen nun die Verhältnisse bei der Phosgenintoxication; die Beziehungen sind aus dem Grunde um so innigere, weil wir annehmen müssen, dass die toxische Nachwirkung des Chloroforms im Körper zum grösseren Theile auf der Bildung von Abbauproducten derselben beruht, welche mit denen des Phosgens jentisch sind, das ebenfalls selbst intermediär als ein solches Umwandlungsproduct des Chloroforms auftreten kann. Dadurch wird abcr das ganze Bild dieser Vorgänge ein sebr wenig scharfes, da diese auftretenden anatomischen Läsionen weder für das Chloroform noch für das Phosgen charakteristisch sind, sondern sich bei ciner grossen Anzahl toxischer Substanzen finden, welche von aussen her auf den Organismus einwirken liönnen, oder im Verlaufe von allerlei Krankheitsvorgängen in ihm entstehen liönnen und welche

1) Nothnagel, Fettdegeneration der Organe bei Aether und Chloroform. Berliner klin. Woohenschr. 1886. No. 4.

2) Bouchard, litude expérimentale sur la mort qui succide a l'injection sous-cutanće de chloroforme sur les aminaux. Gazette hebdomadaire médicale. 1886. No. T. p. 104.

3) Laborde, Centralbl. f. klin. Med. 1886. S. 660.

4) T'oth, Centralbl. f. klin. Med. 1888. S. 241.

5) Ungar, Ueber die tödtliche Nachwirkung der Chloroforminhalation. Vicrteljahresschr. f. gerichtl. Med. N. F. Bd. 47. S. 98.

6) Strassmann, Ueber die tödtliche Nachwirkung des Chloroforms. Virchow's Archiv. Bd. 115. S. 1.

7) Osiertag, Die todtliche Nachwirkung des Chloroforms. Virchow's Arehiv. 13d. 118 . H. 2.

8) Thiem und Fischer, Ueber tödtliche Nachwirkung des Chloroforms. Virehow's Archiv, Bd. 118. H. 2. S. 250.

9) Fraenkel, Virchow's Archiv. Bd. 127. S. 381 und Bd. 129. S. 254.

10) l'ozzi, Do lavaleur des altérations du rein consecutives aux corps fibreux de l'uterus. Annales de gynícol. Juli 1894.

11) Bastianelli, Boll. de la societi I Iancisiana degli ospedali di Roma. 22. V1. $1890, p, 325$.

12) Kast und Mester, Stoffwechselstörungen nach lango dauernder Chloroformnarkose. Zeitsehr. f. klin. Med. XVlll. 1891. S. 469.

13) Ajello, Sullo alterazioni organiche dependenti della narcosi chloroformica. Clinica chirurgica. 1896. No. 8. 
eine Alteration der Stoffwechselvorgänge bedingen, deren sichtbarer $\Lambda$ usdruck eben in diesen allgemeinen Erscheinungen der fettigen Degeneralion vorliegt.

Was nun in den Einzelheiten die Entwickelung des histologischen Befundes anbetrilft, so sollen an der lfand der mikroskopischen Präparate die einzelnen Stadien für die verschiedenen Organe und zwar nacheinander für die Nieren, die Leber und das Myocard, dargestellt werden.

Betrachtet man einen Schnitt aus der Niere eines Thieres, das ungefähr 24 Stunden nach der Phosgeninhalation zu Grunde gegangen ist, so findet sich Folgendes: 1)io Glomeruli sind deutlich vorgrössert, die Gefässschlingen derselben sind gedelint und mit normal aussehenden Blutkörperchen angefüllt, das Glomerulusepithel zeigt keine Veränderungen, der Glomerulusraum ist mit rothen Blutkörperchen ausgefüllt, welcho durch Diapedese dorthin gelangt sind. Weder in der Rindenschicht noch in der Markschicht zeigen die Epithelien der Nierencanälehen Veränderungen, nur Jier und da sind sie etwas comprimirt durch die Erweiterung der ihnen benachbarten Blutgefässcapillaren; an anderen Stellen sind die Canälchen erweitert und vollgestopft mit Blutkörperchen, die theils frei liegen, theils zu Blutkörperchencylindern verschmolzen sind. In dem Maasse, wie diese dem Hilus der Papille näher liegen, nimmt ihre scharfo Begrenzung ab und sie verwandeln sich in homogene, gelbliche, stark lichtbrechende Cylinder (wachsige Degeneration). Im interstitiellen Gewebe sind die Gefässcapillaren erweitert, und es finden sich, namentlich an der Grenze von Rinde und Mark, zahlreiche streifenförmige Hämorrhagien.

Tritt der Tod nach etwa 5 Tagen ein oder tödtet man ein 'Thier nach dieser Zeit, so bietet sich das folgende Bild: Die Glomeruli sind zom 'Theil vergrössert und es findet sich eine Nekrose und Desquamation der Capillarepithelien, derart, dass mitten im Glomerulus oder an seiner Peripherie Gefässsschlingen hervortreten, deren Epithel abgestossen und in homogene, helle Schollen verwandelt ist. An anderen Stellen ist der nekrotisirende Process noch weiter gegangen und der Glomerulus ist geschrumpft, eine deutliche Kernfärbung ist in ihm nicht mehr vorhanden, indem auch das Epithel der Bow man'schen Kapsel degenerirt. Auch das Épithel der Harncanälchen, insbesondere in den gewundenen $A$ bschnitten, zoigt Zellnekrosen. Die Kerne sind nicht mehr distinct färbbar und das Protoplasma ist in eine lörnige homogene Masse, in welcher die Zellgrenzen verwiseht sind, verwandelt. Stellenweise sind auch die Zellen vergrössert und trübc. Das Lumen der Canälchen ist erweitert, stellenweise leer, stellenweise mit hyalinen und körnigen Massen angefüllt, welche von der Desquamation der Epithelien herrühren. Das intertubuläre Bindegewebe tritt deutlich hervor. Vereinzelt finden sich auch Rundzellen im intertubulären Bindegewebe. Dieser Process kann soweit gehen, dass die nekrotischen Lpithelien oder deren Unıwandlungsproducte mit dem interstitiellen Gewebe und den Gefässwänden verschmelzen zu körnigen oder hyalinen Schollen, welchen die ursprüngliche Structur abhanden gokommen ist.

Hand in Hand mit diesen nekrobiotischen Vorgängen verläuft die Bildung der Fetttröpfchen in versehiedenster Grösse und Lage. Diese Darstellung ist ganz knapp und summarisch, aber es würde zu weit führen, auf dio Einzelheiten einzugehen, da dabei einerseits vieles Bekannte wiederholt werden müsste und eine ganze Reihe schwieriger Fragen, wie Herkunft, Bildungsweise, Beschaffenheit der l'etttröpfchen, discutirt werden müssten. So viel lässt sich aber aus dem Vorhergehenden folgern, dass die $\Lambda$ lteration der Nieren mit acuten Entzündungserseheinungen einsetzt, und dass sich an diese nekrotische Vorgänge anschliessen, welche ein mannigfaltiges Bild der entstehenden secundären Degenerationen, hyaline, hydropische, wachsige und fettige Degeneration, liefern können, von denen die letztere sich als die persistenteste erweist und im Fortschreiten des Processes in Concurrenz mit den reparativen Processen der Bindegewebsneubildung immer mehr in den Vordergrund tritt. 
Was die Leber anbetrifft, so ist das erste Stadium dadurch charakterisirt, dass die Capillaren stark erweitert und mit Blut gefüllt sind, dadurch werden die Leberzellstränge zusammengepresst, deformirt und auseinandergedrängt. In den Gefässschlingen der Lebercapillaren finden sich vielfach braunrotbe Pigmentkörner, welche von dem Untergang rother Blutkörperchen herrühren und diese Pigmentkörnchen liegen auch zahlreich im periportalen Bindegewebe und zwischen den Leberzellen. Im zweiten Stadium tritt die fettige Degeneration zunächst in den centralen Zonen der Acini deutlicher hervor, indem dort die grösseren Fetttröpfchen liegen. Vielfach sind die Kerne der Leberzellen zu Grunde gegangen und die Zellstructur weitgehend alterirt, in der Peripherie dagegen, wo die Degeneration weniger weit fortgeschritten ist, zeigen die Zellen vielfach noch ihre normale Form, wenn auch dort die Zellkerne schon vielfach zu Grunde gegangen sind. Das periportale Bindegewebe ist trübe und zellarm. Der Process schreitet in der Weise weiter, dass die fettige Degeneration sich über das ganze Organ ausdehnt, aber nicht überall gleichmässig intensiv ist. In einzelnen Leberzellen finden sich Fetttröpfchen, welche Protoplasma und Zellkern zur Seite drängen; oft ist dadurch die Zelle vergrössert und kugelig an Stelle der normalen polygonalen Form, aber sie besitzt noch einen mondsichelförmigen Protoplasmaantheil, in welohem der Kern liegt. In anderen Leberzellinseln, meist solchen im Centrum der Acini, ist die Degeneration oft soweit fortgeschritten, dass die zelligen Elemente zerstört sind. Es finden sich dort lörnige Herde mit Fetttröpfchenmassen, in welchen die geformten Elemente zu Grunde gegangen sind, sodass dort die Fetttröpfchen und Pigmentlörner frei in den coagulirten Massen liegen, welcho das feine Bindegewebsstroma deutlicher hervortreten lassen.

Im Myocard treten die Veränderungen langsamer hervor und zeigen sich zuerst an den Papillarmuskeln. Es finden sich einzelne Muskelfasern, welche ihre Querstreifung verloren haben und welche weiterhin in homogene, hyaline und durchscheinende Schollen, die fragmentirt sind, sich umwandeln können. Unmittelbar neben diesen degenerirten Fasern finden sich wieder andere, die normal sind. Es kommt also nicht zu einer diffusen fettigen Degeneration, sondern dieselbe tritt in Form kleiner Herde auf. Im Allgemeinen bewahren die degenerirten Fasern ihre Form, ja lassen sogar vielfach noch ihre Querstreifung und ihre Kerne erkennen, sie sind nur breiter als die normalen und ron feinen Fettkörnchen durchsetzt. Auch im Endocard, insbesondere an den Aortenklappen, finden sich entzündliche Veränderungen, welche einer subacut verlaufenden Endocarditis entsprechen.

Auch was die Alterationen der Lungen anbelangt, so sind die hier auftretenden Veränderungen hinsichtlich des anatomischen und histologischen Bildes keine specifischen, sondern solche, wie sie sich bei ätzenden irrespirablen Gasen in mehr minder grossem Umfange einzustellen pllegen. Es entsteben zunächst Zellnekrosen des respiratorischen Epithols, an welche sich eine Iixsudatbildung mit consecutiver Fibrinausscheidung anschliosst, kurz das Bild einer Bronchopneumonie bei längerem Verlaufe, eines acuten Emphysems mit terminalem Oedem bei rasch tödtlich endendem Verlaufe; diese im Einzelnen darzustellen, ist wohl überflüssig, da es sich hier um allgemein bekannte Dinge handelt.

Entsprechend der Intensität der Aetzwirkung verläuft die Reparation dieser Schädigungen der Lungen, wie auch die klinischen Fälle bestätigen, sehr langsam, und es finden sich in solchen Fällen ausgedehnte bindegewebige Narbenzüge, die vielfach zu tiefen Einziehungen der Jungenpleura lühren. Es ist ersichtlich, dass der langsame Verlauf dieser Entzündungserscheinungen und ihrer bindegewebigen Reparation richt nur durch die Störung der normalen Function der Lunge für das Individuum von Bedeutung ist, sondern auch die Möglichkeit bietet zu einer Secundärinfection mit Tuberculose oder zur Activirung eines latenten tuberculösen Ferdes. Aber auch davon abgesehen erweisen sich die Schädigungen des Respirationstractus als äusserst 
Ueber die Einwirkung des Phosgens auf den menschlichen u. thierischen Körper. 125

hartnäckige, welche - beim Menschen - durch langwierige Pneumonien und verminderte Resistenz der Lungen gegen spätere Schädigungen und Infectionen solche Individuen für lange Zeit oder vorzeitig dauernd arbeitsunfähig machen können.

An der Bekämpfung der Schädigungen der Arbeiter, welche in ihren Betrieben möglich ist, hat die chemische Industrie selbst ein grosses wirthschaftliches Interesse; dazu kommt, dass in einem technisch vervollkommneten Betriebe, der mit möglichst hoher Ausbeute zu arbeiten bestrebt ist, die Schädlichkeiten sich von selbst verringern, da der Arbeiter vielfach kaum mehr mit den Stoffen in Berührung kommt. Was weiterhin geschehen kann, um durch Erkenntniss der schädlichen Wirkungen der in der T'echnik verwandten Substanzen eine immer weitergehende l'rophylaxe durchzuführen, das wird die chemische Grossindustrie, der hierzu die Anregungen und Bedürfnisse viel näher liegen und alle Hülfsmittel viel reichlicher zu Gebote stehen, wie jeder anderen Instanz, ebenfalls $z u$ leisten bestrebt sein, und so waren os auch in erster Ijinie deren praktische Interessen, welche die vorstehende Untersuchung und ihre Mittheilung veranlassten. 